\title{
The Spin-2 Equation on Minkowski Background
}

Florian Beyer, George Doulis, Jörg Frauendiener, and Ben Whale

\begin{abstract}
The linearised general conformal field equations in their first and second order form are used to study the behaviour of the spin-2 zero-rest-mass equation on Minkowski background in the vicinity of space-like infinity.
\end{abstract}

\section{Introduction}

In [5], a completely novel finite representation of space-like infinity was proposed. In this setting, space-like infinity $i^{0}$ "blows up" to a cylinder $I$ and, consequently, asymptotically flat initial data become regular near $i^{0}$ and can be prescribed on generic space-like Cauchy surfaces, i.e., the use of hyperboloidal hypersurfaces may ultimately be unnecessary [8]. It turns out that, in this representation, Friedrich's general conformal field equations (GCFE) acquire a very simple form and the cylinder becomes a total characteristic of the system in the sense that there are no radial derivatives in the equations restricted to $I$. In addition, the coordinate location of null infinity is now known beforehand and it does not have to be determined during the evolution. All these features, namely the regularity of the initial data, the a-priori

Florian Beyer

Department of Mathematics and Statistics, University of Otago, P.O. Box 56, Dunedin 9010, New

Zealand e-mail: fbeyer@maths.otago.ac.nz

George Doulis

Department of Mathematics and Statistics, University of Otago, P.O. Box 56, Dunedin 9010, New

Zealand e-mail: gdoulis@maths.otago.ac.nz

Jörg Frauendiener

Department of Mathematics and Statistics, University of Otago, P.O. Box 56, Dunedin 9010, New

Zealand e-mail: joergf@maths.otago.ac.nz

Ben Whale

Department of Mathematics and Statistics, University of Otago, P.O. Box 56, Dunedin 9010, New

Zealand e-mail: bwhale@maths.otago.ac.nz 
fixed finite coordinate location of null infinity, the fact that in the Minkowski case the entire physical space-time can be covered by one computational domain, and the extremely simple form of the evolution equations (especially on the cylinder), make the general conformal field equations suitable for numerical manipulations. However, as expected, the intrinsic system of evolution equations on the cylinder degenerates at the interface of the cylinder $I$ with null infinity. In general, the solutions generate logarithmic singularities at these regions which are expected to travel along null infinity and spoil its smoothness, making it impossible to read-off the gravitational radiation at $\mathscr{I}^{+}$. The way out of this problem is to prescribe initial data that respect the regularity conditions proposed in [5].

In this short contribution, we use Friedrich's general conformal field equations to evolve generic asymptotically flat initial data near space-like infinity. We begin our endeavour from the simplest possible case: linearised gravitational fields on a Minkowski background. Although simple, this "toy model" encapsulates most of the crucial characteristics of the full non-linear system described above. The initial data are evolved as close as possible to the ill-behaved regions $I^{ \pm}$and study the behaviour of the numerical solutions there. This procedure is carried out twice by using the linearised general conformal field equations in their first and second order form. Analytically the two approaches are equivalent, but their numerical implementation could very well differ. The latter statement is partly based on the claim made in [6] that writing the equations of general relativity as a system of second order PDE's is more advantageous numerically than writing them as a system of first order PDE's. According to [6], numerical simulations based on second order PDE's have better numerical accuracy and avoid the appearance of spurious waves travelling against the characteristic curves.

\section{The spin-2 equations}

We use the spin-2 zero-rest-mass equation for a totally symmetric spinor field $\phi_{A B C D}$ in the 2-spinor formulation, i.e. $\nabla_{A^{\prime}}{ }^{A} \phi_{A B C D}=0$, to model linearised gravitational fields on a Minkowski background. Taking the components of the above expression, decomposing the five independent components $\phi_{k}$ of the spin- 2 into harmonic modes $(l, m)$, introducing coordinates $(t, r, \theta, \varphi)$ and an adapted spin-frame on the cylinder, the above spin-2 equation splits into the eight coupled equations (for details see [1])

$$
\begin{aligned}
& \left(1-t \kappa^{\prime}\right) \partial_{t} \phi_{k}+\kappa \partial_{r} \phi_{k}-\left(3 \kappa^{\prime}-(5-k) \mu\right) \phi_{k}=\mu c_{k} \phi_{k-1}, \quad k=1: 4, \\
& \left(1+t \kappa^{\prime}\right) \partial_{t} \phi_{k}-\kappa \partial_{r} \phi_{k}+\left(3 \kappa^{\prime}+(k+1) \mu\right) \phi_{k}=-\mu c_{k} \phi_{k+1}, \quad k=0: 3,
\end{aligned}
$$

where $\kappa(r)=r \mu(r), \mu(0)=1, c_{k}=\sqrt{l(l+1)-(2-k)(1-k)}$, and ' denotes differentiation with respect to $r$. Equations (1), when appropriately combined, split into three constraint equations and a symmetric hyperbolic (in the domain $|t|<\kappa^{-1}$ ) system of five evolution equations, see [1]. 
By differentiating the above spin-2 equation, one can derive (see [4]) the second order spin-2 wave equation $\square \phi_{A B C D}=0$. Decomposing again the components of the spin-2 field into harmonic modes and introducing coordinates $(t, r, \theta, \varphi)$, the spin-2 wave equation splits into a hyperbolic (in the domain $|t|<\kappa^{-1}$ ) system of five wave equations

$$
\begin{aligned}
\left(1-t^{2} \kappa^{\prime 2}\right) \partial_{t t} \phi_{k} & -\kappa^{2} \partial_{r r} \phi_{k}+2 t \kappa \kappa^{\prime} \partial_{t r} \phi_{k}+2 r \kappa \mu^{\prime} \partial_{r} \phi_{k} \\
+ & 2\left[(2-k) \kappa^{\prime}-t\left(\kappa^{\prime 2}+r \mu^{\prime} \kappa^{\prime}-\frac{1}{2} \kappa \kappa^{\prime \prime}\right)\right] \partial_{t} \phi_{k} \\
+ & {\left[(2-k)\left(\kappa \kappa^{\prime \prime}+(1-k) \kappa^{\prime 2}\right)+k(5-k) r^{2} \mu^{\prime 2}\right] \phi_{k} } \\
& =-\mu^{2} c_{k}^{2} \phi_{k}-r \mu \mu^{\prime}\left((4-k) c_{k} \phi_{k+1}+k c_{k-1} \phi_{k-1}\right) .
\end{aligned}
$$

Notice that the domain of hyperbolicity for both sets of equations is the same, namely $|t|<\kappa^{-1}$. Thus, as expected, in both approaches the equations degenerate at $(t, r)=( \pm 1,0)$, i.e. at the regions $I^{ \pm}$where null like infinity $\mathscr{I}^{ \pm}$meets the cylinder.

In order to compute the same solution as with the first order system (1) we have to use all the available information from the first-order system to determine initial and boundary conditions for the second order system (2). In [4] it was shown that the two formulations are equivalent provided the initial and boundary data for the second order system are determined from the first order system.

\section{Numerical results}

The PDE systems (1), (2) are discretised according to the method of lines. An equidistant grid on the computational domain $D=[0,1]$ is used to discretise the spatial coordinate $r$. The spatial derivatives are approximated by summation by parts (SBP) finite difference operators [7]. The boundary conditions are implemented with

\begin{tabular}{|c|cc|rc|}
\hline & \multicolumn{2}{|c|}{$\phi_{0}$} & \multicolumn{2}{c|}{$\phi_{4}$} \\
\hline Grid & 1st order & 2nd order & 1st order & 2nd order \\
\hline 50 & -25.2218 & -27.6006 & -11.1643 & -12.3418 \\
\hline 100 & -29.3956 & -31.5941 & -13.9924 & -15.1743 \\
\hline 200 & -33.7109 & -35.6075 & -16.9978 & -18.1782 \\
\hline 400 & -38.1000 & -39.6068 & -20.1075 & -21.2850 \\
\hline
\end{tabular}

Table 1 The logarithm of the normalized $l^{2}$ norm of the absolute error $E, \log _{2}\left(\|E\|_{2}\right)$, between the exact solution and the solutions computed from the 1st-order system (1) and the 2nd-order system (2) at time $t=1$.

a very simple, but highly efficient, simultaneous approximation term (SAT) penalty method [3]. The temporal integration is based on a standard explicit fourth order Runge-Kutta scheme. The code has been written form scratch in Python. 
The comparison of the numerical properties of the two approaches is based on their ability to reproduce a specific family of exact solutions of the spin-2 equation developed in [1]. As was shown in [1,4], the critical sets $I^{+}$, located at $t=1$, can be reached in both approaches without loss of the expected 4th order accuracy; in addition, the constraint quantities are preserved to sufficient accuracy during the evolution. A comparison of the accuracy with which the two approaches numerically reproduce the exact solution is shown in Tab. 1. Better accuracy was achieved in the second order formulation, a result that confirms the first claim in [6]. The second claim made therein that the spurious waves disappear in the second order case is also confirmed as the high frequency features disappear in the convergence plots of the second order formulation, see [4].

\section{Conclusion}

In this work, two distinct approaches to the linearised general conformal field equations were developed and subsequently implemented numerically. In both approaches we managed to reach without loss of accuracy the ill-behaved region $I^{+}$. It is principally not possible to go beyond $I^{ \pm}$since the equations loose hyperbolicity. A possible way to resolve this problem is presented in [2]. We have also shown that the second order formulation of the spin-2 equation leads to a better accuracy by a factor of 3-4 and the spurious waves travelling against the characteristics disappear, confirming the claims made in [6].

\section{References}

1. Beyer, F., Doulis, G., Frauendiener, J., Whale, B.: Numerical space-times near space-like and null infinity. The spin-2 system on Minkowski space. Class. Quantum Grav. 29, 245013 (2012)

2. Beyer, F., Doulis, G., Frauendiener, J., Whale, B.: Linearized gravitational waves near spacelike and null infinity. (this volume), arXiv:1302.0043 (2013)

3. Carpenter, M.H., Gottlieb, D., Abarbanel, S.: Time-stable boundary conditions for finitedifference schemes solving hyperbolic systems: methodology and application to high-order compact schemes. J. Comp. Phys. 111, 220-236 (1994)

4. Doulis, G., Frauendiener, J.: The second order spin-2 system in flat space near space-like and null-infinity. to appear in Gen. Relativ. Gravit., arXiv:1301.4286 (2013)

5. Friedrich, H.: Gravitational fields near space-like and null infinity. J. Geom. Phys. 24, 83-163 (1998)

6. Kreiss, H.O., Ortiz, O.E.: Some mathematical and numerical questions connected with first and second order time-dependent systems of partial differential equations. Lect. Notes Phys. 604, 359-370 (2002)

7. Mattsson, K., Nordström, J.: Summation by parts operators for finite difference approximations of second derivatives. J. Comp. Phys. 199, 503-540 (2004)

8. Moncrief, V., Rinne, O.: Regularity of the Einstein equations at future null infinity. Class. Quantum Grav. 26, 125010 (2012) 